\title{
Pan-neuro: interactive computing at scale with BRAIN datasets
}

Ariel Rokem ${ }^{1}$, Benjamin Dichter ${ }^{2}$, Chris Holdgraf ${ }^{3}$, Satrajit Ghosh ${ }^{4}$

1. Department of Psychology and eScience Institute, University of Washington

2. CatalystNeuro

3. International Interactive Computing Collaboration

4. McGovern Institute for Brain Research, Massachusetts Institute of Technology

\section{Abstract}

New technical and scientific breakthroughs are enabling neuroscientific measurements that are both wider in scope and denser in their sampling, providing views of the brain that have not been possible before. At the same time, funding initiatives, as well as scientific institutions and communities are promoting sharing of neuroscientific data. These factors are creating a deluge of neuroscience data that promises to provide new and meaningful insights into brain function. However, the size, complexity, and identifiability of the data also present challenges that arise from the difficulties in storing, accessing, processing, analyzing, visualizing and understanding data at large scale. Based on their successful adoption in the earth sciences, we have started adopting and adapting a set of tools for interactive scalable computing in neuroscience. We are building an approach that is based on a combination of a vibrant ecosystem of open-source software libraries and standards, coupled with the massive computational power of the public cloud, and served through interactive browser-based Jupyter interfaces. Together, these could provide uniform universal access to datasets for flexible and scalable exploration and analysis. We present a few prototype use-cases of this approach. We identify barriers and technical challenges that still need to be addressed to facilitate wider deployment of this approach and full exploitation of its advantages.

\section{Introduction}

\section{Big data neuroscience}

Neuroscience is at the cusp of a new era of Big Data research. This shift is happening thanks to a confluence of factors: (1) technical and scientific breakthroughs that have enabled large-scale data collection; (2) funding initiatives, such as the BRAIN Initiative, which have supported the rapid evolution of these technologies and their application to the study of many different research questions; and (3) changing attitudes of researchers who, thanks to data-sharing 
initiatives such as the Allen Institute for Brain Science, are starting to realize the benefits of data sharing and are more willing to provide raw large-scale datasets for use by other researchers. Taken together, these factors are allowing researchers to gain access to unprecedented amounts of information about the structure and function of the brain, from multiple different models and systems and at multiple different scales.

Large and complex datasets, with transformative implications to our understanding of the brain -- are simultaneously emerging across multiple neuroscience subfields [1]. Examples driven by recent technological breakthroughs are salient in neurophysiology where new optical imaging techniques such as SCAPE microscopy enable volumetric imaging of brain tissue over time. High density Neuropixel probes have $\sim 1,000$ recording sites and can record from about 250 neurons at the timescale to observe individual action potentials, but at the cost of unprecedented data volume, with projects producing raw data at the scale of 100 s of TB. Raw Neuropixel datasets have already started to be published publicly [2].

Human neuroimaging is also experiencing developments in technology that are driving increased resolution of the measurement and concomitant increases in data volume. At the same time, human neuroscience demonstrates that targeted funding and sociotechnical trends are also driving increases in the widespread availability of very large datasets. For example, through concerted efforts to collect and aggregate large-scale datasets with data from hundreds or even thousands of individuals. A paragon of this kind of data collection is the $\mathrm{NIH}$-funded Human Connectome Project (HCP), which provides high-quality MRI data from more than 1,200 young healthy individuals, coupled with behavioral and genetic information[3]. At this scale, the volume and complexity of the data are staggering: over 80 TB of data stored in $\sim 15 \mathrm{M}$ files. But this is just the beginning. Several ongoing studies are currently amassing datasets with many thousands of individuals: The Healthy Brain Network study[4] and Adolescent Brain Cognitive Development study[5] will each collect cross-sectional and longitudinal data from approximately 10,000 children during their development, together with detailed information about their cognitive and mental health development. The UK Biobank provides MRI measurements from about 43,000 participants and will eventually provide data from approximately 100,000 individuals[6]. Many other projects are also collecting very large datasets on a variety of clinical populations. These trends are not likely to abate, as the field is becoming increasingly aware that large samples are crucial for reproducibility and for accurate inferences about the role of brain structure and function in complex behaviors and in mental and neurological health[7].

These datasets are already having a profound impact on neuroscience, as data-driven methods are employed to illuminate brain function and structure in new and important ways. At the same time, the deluge of data is generating new challenges, as researchers struggle to find, store, manage, analyze and interpret the data. This is because Big Data compels a paradigm shift in computational methodology. For example, electrophysiological recordings from thousands of single units or MRI measurements at the scale of hundreds of individuals cannot be easily copied and moved. 


\section{Cloud computing}

The challenges described above are common in many research fields, as well as in commercial applications of data analytics, and a new generation of tools is evolving to address the challenges of these Big Data. These tools use cloud computing as their primary paradigm. In this paradigm of computing, files containing the data are stored off-site in a server facility and are rarely copied or moved from a central storage location. Computing is done on processing units that are physically close to the data, avoiding costly and even prohibitive communication delays [8-10]. The physical infrastructure -- computers and storage devices that are used -- may be replaced in a manner that does not impact the end users. Typically, cloud computing is associated with the services provided by the so-called public cloud: commercially-available vendors, such as Amazon Web Services, Google Cloud Platform, Microsoft Azure and others that provide Infrastructure as a Service (laas).

Cloud computing offers flexibility, scalability, portability, and reliability, as well as accessibility, and the cloud paradigm has been broadly adopted in many business use-cases [11]. For the same reasons, it holds much promise in scientific application as well, but the broad adoption of cloud computing in neuroscience is hampered by several gaps, which still need to be filled to effectively harness these tools in the context of neuroscience research:

(1) User experience: broad adoption of cloud computing would require the development and dissemination of flexible tools for data visualization and exploration of neuroscience data that provide similar user experiences, regardless of whether they are run locally or in the cloud.

(2) Deployment: Easy deployment of these tools to a variety of computational resources would help accommodate the wide range of users.

(3) Scalability: It should be as straightforward and as fast to run an analysis on one brain as it is on 1,000 brains, as easy to analyze the responses of a single cell as it is to analyze responses from a population of many thousands.

Given the sizes of many emerging neuroscience datasets, significant consideration needs to be placed on the movement and storage of data as well. Taken together, addressing these factors will significantly lower the barriers to adoption of cloud computing in neuroscience research and broaden the potential research community that can use these datasets.

\section{Pan-neuro and the importance of interactive computing}

We propose that the fundamental issues described above -- user experience, flexible deployment and scalability - can be addressed by building upon a set of existing and evolving open-source tools, and through community-based practices for their use. We take inspiration from our collaborators working in the Earth Sciences, where a similar set of challenges has emerged at a high level of intensity over the last few decades. Specifically, we propose to extend 
the approach taken by a particular community, the Pangeo community [12], into neuroscience. Taken together, the tools and practices that we propose to adopt constitute what we call "Pan-neuro" -- an approach to interactive computing at scale with large neuroscience datasets.

The fundamental toolset used in Pangeo includes datasets that are shared through a centralized storage mechanism and attached to proximally-located compute nodes (e.g., in the same data center, whether in a commercial cloud computing provider, one run by a university IT organization, or via national infrastructure, such as XSEDE), together with a set of open-source libraries that are used to analyze the data.

The front-end for Pangeo-deployed systems is provided through a Jupyter interface. Project Jupyter builds open source tools, protocols, and standards for interactive computing in data science [13], including a widely adopted computational notebook format and user interfaces to run this format. However, the project has a much broader scope than just the notebooks: it builds interoperable, modular infrastructure that spans the interactive computing workflow--from low-level kernel implementations to user interfaces, to cloud infrastructure management. Jupyter tools have been widely recognized as a crucial tool for data science work, including a 2018 ACM Software Systems award.

The interactive nature of computing in Jupyter is essential to the process of computational scientific discovery with this tool. This is because it enables a rapid cycle of writing and running software -- exploring intermediate results of computation and refining the computation along the way. This runs counter to the idea that one might have about scientific computing, where an analysis plan developed a priori is mapped to the data, and then executed once, with the results appearing on the other side. In contrast to software engineering in industrial applications, research and data analysis often demand active exploration of the data, complementing and augmenting strict hypothesis-driven analysis [14,15]. Interactive computing entails an altogether different approach than that taken in many industrial applications, opening up possibilities that are not accessible in other computational modes [16].

JupyterLab is a recent evolution of the Jupyter Notebook interface that provides a more flexible and modular user interface. It serves as a web-based front-end for Pangeo systems, allowing users to create and run computational notebooks, including code, visualizations and text blocks. JupyterLab is extensible, and can be customized with tools to directly interact with data stored in cloud computing, view the contents of data files, and edit them. Because of this flexibility, rich interactive interfaces can be created for domain-specific data formats. For example, a custom viewer for the commonly used geoscience GeoJSON format provides a map view that displays the annotations stored in the GeoJSON file and allows adding annotations directly to the map.

JupyterHub provides authenticated access to the interactive computing environments and backend infrastructure that users access via a web portal. It provides the mechanisms that support launching notebooks for use by multiple users. It is designed to be flexible and scalable, allowing administrators of these systems to select and mix a variety of computational resources, programming language kernels, front end user interfaces, or connections to datasets. It is vendor- and cloud-agnostic, and can be used in conjunction with other Jupyter tools such as JupyterLab, or as a standalone service for hosting interactive sessions in general. 
Jupyter's modular design allows it to integrate with many libraries for numerical computation, such as the Dask distributed computing library[17]. In cloud-computing environments, this integration uses Kubernetes, a container orchestration system originally designed by Google engineers, and now available as open-source software that runs on all major cloud infrastructure[18]. Kubernetes allows multiple users of a JupyterHub deployed to a cloud-based cluster to scale computations to hundreds or even thousands of compute nodes, as needed, elastically scaling down when the work is done.

The uniform set of open-source tools -- Jupyter, Kubernetes, Dask, etc. -- provides a good basis for the evolution of a vibrant community of practice in geoscience that encompasses researchers working in various subfields of geoscience. The global access to Pangeo-deployed hubs through the internet provides a basis for collaborations that transcend institutional and even national boundaries.

In what follows, we will provide a few prototype examples of the Pan-neuro approach that we have deployed and developed. Then, we will discuss the gaps that still need to be filled for this approach to reach its full potential, and propose a few next steps that we and others can take to achieve progress towards the vision of interactive scalable computing with large neuroscience datasets.

\section{Pan-neuro in action: a few prototype examples}

Widespread dissemination and adoption of the Pan-neuro approach will require a smooth user-experience that can only be achieved by considering the entire software ecosystem around specific use-cases. We have deployed systems like the ones that we envision in a few use-cases that we describe below. The range of these use-cases demonstrates the versatility of the basic tool-set.

\section{Use-case 1: Collaborative hub for team science}

A JupyterHub deployed in cloud computing infrastructure can serve as the basis for collaborative work within a research group, or in the context of larger team science projects. One such context in which we have deployed this kind of system includes a BRAIN Initiative U19 Brain Circuits Program project, led by Beth Buffalo at the University of Washington, and includes collaborating teams from several other institutions (NINDS 5U19NS107609-03). The project collects multi-channel electrophysiological recordings from human and non-human primates, while they are performing complex learning tasks. In human subjects, data are obtained from epileptic patients undergoing monitoring with ECoG. In the non-human primate model, recordings are obtained from multiple different locations, including frontal cortex and hippocampus. Datasets are uploaded by participating groups into commercial cloud object storage in a uniform light-weight BIDS-inspired format [19]. The hub and data are kept private, with authentication using GitHub's OAuth. Scalability is achieved through use of the Dask library, primarily in mapping Python-based signal processing and machine learning software [20,21] to these large recordings. Ultimately, the goal is to interface with the data in the Neurodata Without 
Borders (NWB) format, but intermediate formats (Matlab HDF5, primarily) are also used, while some of the groups are still not fully set up to use NWB yet. This hub was originally deployed on the Google Cloud Platform, but was recently migrated to Amazon Web Services (AWS), to facilitate interoperability with storage of some of the experimental raw data, which is in AWS. In this case, many of the scientists were unfamiliar with Python and with the use of Jupyter, so hands-on training of scientists on use of the tools was a required part of introducing the tools.

In another collaboration, funded through the BRAIN Initiative Informatics program (1RF1MH121868-01; https://autofg.org/), we are analyzing publicly available datasets of human MRI data, primarily from the Human Connectome Project [3]. In this case, the analysis pipelines [22] have already been hardened to the degree that they just need to be deployed at scale on these datasets. In these cases, interactive computing is less essential, and efficiency of deployment at scale at low cost is a higher priority. For this kind of deployment, we use tools that we have developed for packaging of well-defined computation in docker and automated deployment to AWS Batch [23]. However, the outputs of these analysis pipelines are made available for further statistical analysis, exploration and visualization in a JupyterHub deployed in Azure. Here as well, training of researchers was essential. One interesting observation from this work is that adoption of the new tools may correlate inversely with level of prior experience. New research students faced with a JupyterHub happily jump into it and start rapidly being productive on the platform, while researchers that are not used to the web-based tools and have already established productive desktop-bound workflows are more resistant to the change. In these cases, the ease of access through the browser does make the initial on-ramp for new researchers, even those with very little experience in neuroscience data analysis, relatively straightforward.

\section{Use-case 2: Gateway to BRAIN Archives}

As part of the BRAIN Initiative archive DANDI for cellular neurophysiology, we have deployed DANDIHub, an instance of JupyterHub, deployed in AWS. This instance provides access to all the data in the archive (presently at 42TB) over a 100 Gbit connection, and is available to researchers who register on DANDI. We also provide basic demonstration notebooks to enable reusing such data. Many of the datasets have TBs of data, which would be time consuming to transfer, if only a small fraction of the information is required. To address these use cases, we worked with the community to integrate more direct access methods to data stored in Neurodata Without Borders (NWB) HDF5 files. You can now use the "read only s3" (ros3) driver to stream data directly from the $s 3$ buckets of DANDI or any other public s3 bucket, and read only the data needed. Data streaming can be employed in conjunction with NWB Widgets, a package that offers interactive domain-specific visualizations of NWB files within the Jupyter Lab environment. However, these approaches are not yet standardized in the community and effort is required to coordinate across several use-cases. For example, it is not clear how these approaches would be adopted in human neuroimaging, where the commonly-used binary file-formats are not based on the HDF5 format. DANDI is also supporting several different data modalities around cell level information: electrophysiology, optophysiology, and behavioral 
time-series, and images from immunostaining experiments. Having these accessible from a single archive is of significant utility to a scientist. Similar to DANDI and DANDIhub, other BRAIN archives are also developing approaches to bring compute to data stored in the cloud (e.g., BossDB and NeMO).

\section{Use-case 3: Portal for training in data science}

One of the major gaps that web-based interactive computing can help solve is training in data science. In the past, one of the initial hurdles to training in data science was the installation and configuration of software on individual trainees machines. Software Carpentry, a non-profit organization devoted to teaching basic computing skills to researchers, has developed detailed protocols and instructional videos to walk participants in their workshops through the installation process. But individual laptops in the wild are such a variable and moving target that this still typically fails in at least one case for most workshops. Another typical solution is to provide training in a computer laboratory equipped with enough computers for all of the participants, and where hardware and software installation can be guaranteed to be uniform. Some other training courses solved this problem by providing laptops to all of their participants! Cloud-based installation of Jupyterhub systems, including installation of specialized analysis software, allows participants in a training course access to a uniform and preconfigured computational environment [24]. We have used this kind of environment extensively in our training engagements. One example is the Summer Institute in Neuroimaging and Data Science, held at the University of Washington in Seattle, with funding support from NIMH (5R25MH112480-05). The SINDS (also known as "NeuroHackademy", https://neurohackademy.org) is divided into two week-long portions. The first week focuses more on instructional activities. This includes tutorials in a variety of data science topics (programming, machine learning, data visualization, and so forth), with significant hands-on components. It also includes lectures on the application of data science methods in neuroscience. The second week, organized as a hackathon $[25,26]$, focuses on participant-driven activities and primarily on team projects. In this case, when participants log into the hub, it is already equipped with a set of notebooks that instructors have composed for the purpose of the tutorials, as well as all of the software that participants need, and data used in the tutorials. The configuration of the hub is managed through publicly accessible version control, and it can rapidly be adjusted based on instructor or participant requests. For example, to add installation of software or data needed for a particular tutorial or project. Because it is accessible through an internet browser, the JupyterHub also supports remote/online training, such as the one that NeuroHackademy held for $>300$ participants in the summer of 2020 and for 50 participants in summer 2021.

The ABCD-ReproNim course (https://www.abcd-repronim.org/) with funding from NIDA (1R25DA051675-01) is another example of a datascience course focused on a large dataset accessible in the cloud and used a Jupyterhub deployment to teach students how to retrieve and work with such data. In addition, the same container used in the cloud, could be used by students in their local environments to work locally in their compute clusters using the $A B C D$ 
data. This flexibility provided maximum utility to the data as not all computations need to be performed in the cloud and makes the data accessible to those who do have local compute services available.

\section{Barriers to progress and ways to address them}

\section{Cloud-optimized data standards}

The first challenge is the classic data standards challenge: analysis tools require a standardized data schema that specifies the form of data and associated metadata. The standard ensures that all of the necessary metadata is present and allows data from multiple sources to be aggregated together in a machine-readable way. The creation of such a schema is particularly challenging in neuroscience due to the heterogeneity and rapid evolution of data acquisition technologies. Adherence to this schema requires either robust conversion pipelines from existing data formats or modification of acquisition systems to write directly to the standards, which, again, is challenging due to the diversity of the technologies.

In addition, in the context of cloud computing, scalability of both the computing and the storage offers the opportunity for rapid parallel data access, which can accelerate processing of the data [27]. However, this also requires that the data adheres to a format that supports parallel access patterns. There are different file formats that support parallel reads in a way that provides a good fit to cloud computing. In particular, HDF5 files that are used in many scientific computing contexts -- for example, as a storage mechanism for Neurodata NWB files -- can be read by multiple processes simultaneously. Moreover, the ros3 driver enables streaming data directly from AWS S3 buckets, a particularly advantageous mechanism in the context of cloud computing. HDF5 can also be configured in parallel mode, which uses Message Passing Interface (MPI) to enable parallel processes to simultaneously write to the file as well, for other kinds of use-cases.

An example of an emerging standard for analysis-ready cloud-optimized data is the merger of HDF5 and NWB through the use of the ZARR standard [28]. ZARR is a format for the storage of chunked, compressed, $\mathrm{N}$-dimensional arrays. Array data are ubiquitous in neuroscience: time-series of multi-channel recordings, images at various scales, and combinations thereof in high-resolution video both of recorded data, as well as in recordings of complex behaviors. The ZARR standard allows both reading or writing of large array data concurrently from multiple threads or processes. This enables parallelized modes of computing, such as those supported by the Dask software library. This combination (Dask + ZARR) has also been in extensive use in the Pangeo community, to perform scalable computation on large earth-science datasets, and one of the remarkable outcomes of the Pangeo effort has been the migration of important geospatial datasets into analysis-ready cloud-optimized formats (https://pangeo-forge.readthedocs.io/). Adopting the ZARR standard would allow neuroscience to borrow strength from the development efforts that have been happening in those fields. 


\section{Sustainability and considerations for preservation of data}

With the development of higher spatio-temporal acquisition instruments, the size of new data is increasing exponentially, and it may become prohibitively expensive to preserve the data from all experiments indiscriminately. In some domains, such as extracellular electrophysiology and calcium imaging, the preprocessing pipelines compress the data to simpler derivatives that are much smaller in size. These smaller data could be shared instead of the raw data, but we would lose the ability to reprocess the data as the preprocessing software improves and could be locked to the current state-of-the-art and whatever parameters were chosen the time. On the other hand, some preprocessing pipelines such as spectrotemporal decomposition expand the size of the data many times. These computations can be expensive, and it would be convenient to store the intermediate data, particularly in interactive workflows where this preprocessing is the rate-limiting step, but the storage of this data exacerbates the challenge of data preservation.

The increased data volume also makes transport of data challenging both within and out of institutions. In some situations data are sent using tape drives (e.g., fMOST data), while in others data are consolidated locally and only the publishable components are uploaded. Many institutions do not have high-bandwidth networking connecting data acquisition in labs to the outside computational infrastructure, which slows down upload of data. There is thus a need for some form of consensus on common workflows, data to preserve, and a period for sunsetting data.

Currently, the community lacks common protocols to determine what to upload into the cloud in the first place, and also what to retain in the long run. Most neuroscience communities are still figuring out which elements of data to preserve and which workflows to use. For the time being, this necessitates preservation of the raw and derived data, at least in the short term, thus increasing significant demands on storage.

\section{Cost and lock-in of cloud storage}

While storing in the cloud and computing near it has significant advantages, there are several additional considerations. The richness of available data also implies that the analyses have long tails of data use across different neuroscience disciplines and require extended storage. If the current status quo persists, cloud storage cost would be a significant impediment to the use of cloud computing. For example, storage of 1PB of data on AWS simple storage service (S3) costs about $\$ 25 \mathrm{~K}$ per month. For the cost of storing that amount of data on S3 for one year, even with relatively low bandwidth access (S3 provides several tiers of storage, and cost varies based on bandwidth and on access patterns), researchers could store the data in efficient data centers across national infrastructure for 3 years at least, with high bandwidth access speeds to compute resources that are adjacent to the data within the same data center. And this is just a single copy of the data. Archiving in a secondary storage increases the total cost of storage. However, this comparison is further complicated by the fact that academic non-cloud infrastructure is also subsidized through other means. For example, high-performance 
computing at universities is typically funded centrally, in addition to the funding that comes directly from users. Cost calculations become even more complicated when considering that, while getting data into the cloud is typically free, most cloud services charge for sending data out of the cloud, which means that users have to be able to work on the cloud closest to the data. It also requires infrastructure that optimizes where data are stored based on whether data will be added to or just read for analysis. While the use of technologies such as Kubernetes that can be deployed to any cloud vendor should help mitigate vendor lock-in, the current infrastructure choices can easily create a locked-in environment for data storage, which may not be optimal for academic research and makes researchers dependent on the whims of an external entity. Such an entity can choose to increase prices for either storage or data movement, making it even more prohibitive to get data out of a cloud. So far, commercial cloud vendors have taken steps to mitigate these concerns, by providing academic researchers with data egress waivers and providing free storage for large high-value datasets. For example, the Human Connectome Project, several datasets from the Allen Institute for Brain Science, and the BRAIN Initiative OpenNeuro human MRI and DANDI, as well as other BRAIN Initiative datasets, are all stored in Amazon Web Service's Open Data Program.

Coordination and policy development across federal, commercial, and academic entities will be required to create an ecosystem that allows transparent and low-cost data movement that maximises the utility of the data and minimizes control by a single entity. Community-driven tools with open standards and protocols, such as Jupyter, Dask and Zarr can help mitigate the risks for data lock-in by cloud vendors, by reducing the dependency of research efforts on vendor-specific tools.

\section{Neuro-specific analysis tools}

As mentioned above, we extensively use the Dask software library as a basis for scalable computing in large distributed cloud-based systems. Many common analyses in neuroscience can leverage scalable computation that has already been implemented in domain-agnostic tools. For instance, in auditory neuroscience, it is common to compute spectro-temporal receptive fields (STRFs), which identifies the auditory pattern that activates a neural response. This is often an application of regularized linear regression, a commonly-used and domain-agnostic algorithm that has been implemented in Dask-ML, a library of machine learning algorithms that uses Dask for efficient parallel computation. Dask-ML also provides scalable algorithms for classification and clustering, which are also common tools in computational neuroscience. These examples suggest that we can use Dask-ML and similar domain-agnostic data analysis libraries in neuroscience. Increased efficiency and clarity would be achieved by writing software that presents an API based on concepts familiar to computational neuroscientists, and that interfaces with common neuroscience data formats such as NWB and BIDS.

While Dask already supports some numeric computing operations, analysis of neuroscience data also requires implementations of specific computational routines and pipelines that are not yet implemented. An example from earth-sciences is the development of 
XArray [29], which provides an abstraction layer for operations on geospatial data that easily plugs into the Dask/Zarr ecosystem. By handling the domain-agnostic machine learning with Dask-ML and similar tools, and contributing to those tools where appropriate, we will also be creating a shared inter-domain computational infrastructure that can benefit multiple scientific communities simultaneously.

To make these pipelines and their results comply with the FAIR principles [30] would require additional infrastructure that uses provenance to reduce the burden of recomputation of the same operation, and that allows the reuse of data generated by others. Findability of these results would require metadata annotation that would make search possible. This entails that the existing software infrastructure (i.e., Dask) needs to be extended to support these requirements.

\section{Visualization and annotation tools}

Interactive exploration and annotation of neuroscience data is a crucial part of many data analysis steps including quality control[31], delineation of regions of interest or cortical maps[32], and virtual dissections of white matter connections[33,34], manual curation of image segmentation and spike sorting, among others. These platforms need to support interactive exploration and annotation of neuroscience data, such as viewers for the common data file-formats. There are a variety of implementations for browser-based visualizations of neuroscience data [35-38]. However, to make these systems useful, these would need to be extended to: 1) Work with data stored in cloud storage and 2) Seamlessly operate within the Jupyterlab environment. Future work could support many different use-cases. For example, it could enable data annotations, such as drawing of ROIs, and even data editing, as in "virtual dissections", with the option to immediately save annotations and edited data sets back into cloud storage. As in the scalable computing case, one promising direction would be to adapt tools that have evolved in other domains (e.g., Glue [39] or Yt [40], which evolved in different parts of the astrophysics research community), or in domain-agnostic contexts (e.g., datashader [41], a domain-agnostic software library for visualization of very large datasets in the browser). Encouragingly, there is already a vanguard of tools that have been developed specifically for neuroscience $[38,42]$.

\section{DevOps and cloud-native expertise}

One of the phenomena attendant to the rise of cloud computing is the culture of DevOps. This is a set of practices and tools that are commonly adopted in the deployment of cloud-based systems. Generally speaking, devops relies on explicit and declarative configuration of the components of a system (or "Infrastructure as Code", laC), as well as a reliance on automation for rapid incremental change (e.g., continuous integration and continuous deployment). In addition to these mostly technical choices, devops espouses the narrowing of gaps between IT/infrastructure and software development, and the increased communication within organizations. While laC approaches significantly reduce the amount of work needed to deploy 
and maintain complex cloud-based infrastructure, adopting these approaches entails a significant learning curve and requires a new and unique set of tools and skills. These skills are rare and in high demand, making it hard to hire individuals that possess them into research organizations. This means that, in the short term, there will be a skills gap between the cloud-native workflows that scientists will need to do their work, and the kinds of services that universities are equipped to provide. In academic institutions, the adoption of devops culture could also entail a shift in the traditional relationships between research IT, research software development and scientists. This comes with a new risk of lock-in: while the open source tools are available to power platforms like Pangeo, the skills needed to duplicate and run those platforms anew may not always be available, causing research groups to turn towards proprietary "Software as a Service" offerings.

To mitigate this risk, research organizations should invest in training and hiring for cloud-native expertise. In situations where outsourcing cloud deployment may be necessary, this should be done in a manner that maximizes flexibility and choice, and that minimizes the risk of vendor- or service-lock-in. A natural way to achieve this is by choosing services that only utilize open-source, community-led tooling that is supported via a variety of stakeholders, as well as those with a clear commitment to building on open standards and protocols to ensure interoperability between vendors and toolchains.

\section{Sociotechnical support: data sharing, training and incentives}

Some of the most difficult challenges to the adoption of the Pan-neuro approach are sociotechnical in nature. Some of these issues have to do with the publication of experiment data through shared archives, and the effective and equitable use of shared data resources, in a manner that respects the rights of data producers, while also enabling maximal utility from the existing datasets. Clarity about the rights and duties of both data producers and data users should help to address these issues. For example, data sharing mandates coupled with new funding opportunities for reuse of published data have sent a strong signal to the neuroscience community. A cultural shift in the attitudes towards data sharing and its benefits to data producers, data users and the broader community of stakeholders is already underway.

Another challenge of a sociotechnical nature relates to training. The Pan-neuro toolset is different from some of the tools used in the past. For example, while open-source software has made significant inroads in neuroscience, with thriving research communities that rely on these tools, many neuroscientists still use proprietary desktop-bound tools, such as Matlab, for their data analysis. For these researchers to be able to adopt and adapt to the tools proposed for Pan-neuro, they will have to learn how to perform their data analysis using new tools. Accelerated adoption is facilitated by adoption of Python as the teaching language, and the use of Jupyter notebooks in many training courses focused on computational neuroscience and on the use of open data resources, among them the Allen Institute/University of Washington Summer Workshop on the Dynamic Brain and the Neuromatch Academy.

Finally, there is a set of sociotechnical challenges related to providing support for researchers in neuroscience and in other domains to work on tools that serve more than one 
scientific domain. Most current academic organizational structures and current funding incentives do not support this kind of domain-general work. High-quality community-driven software development requires teams of highly skilled individuals that can build and support the software over long time horizons. The skillset and time commitment required for this work does not fit well into academic positions, nor is it often the most direct path to achieve high-profile publications that are incentivized by the traditional academic structure. Therefore, it should be acknowledged from the outset that the traditional academic structure is not ideal to support the development of these tools. While some of the technologies mentioned here would be of considerable sophistication and would merit publications in their own right, building and maintaining the community around these resources requires a large amount of support work such as writing documentation, testing software, and supporting users of the software and developers building upon it. This work constitutes intellectual infrastructure of crucial importance. The work of our geoscience collaborators in Pangeo again demonstrates that building and sustaining a significant and growing community of practice around this toolset is a key element in its success.

\section{Summary and conclusions}

Large shared data sets provided through easy access will become the "digital watering hole"1 of the next decades of research into the brain, providing unique opportunities for research that integrates information across scales and measurements and that brings in researchers from other fields (e.g., mathematical and physical sciences). The BRAIN Initiative in particular, with the new and data-intensive measurement technologies that it is developing; with it's forward-looking data sharing mandates, its extensive cyberinfrastructure in the form of data standards, data archives; and with its plethora of software tools, is providing the basis for the Big Data future of the field of neuroscience, one that is rapidly becoming a Big Data present. To capitalize on these opportunities, we need to provide easy and immediate access to this digital watering hole to a wide range of researchers, through powerful tools that support interactive exploration and scalable analysis. The ecosystem of software tools and technical approaches that the Pangeo project is using and evolving in the earth sciences is a good starting point. However, to harness these tools and the Pangeo approach in neuroscience would require addressing the specific challenges and needs of our field. A coordinated community effort would help to more readily and rapidly unleash the potential of these datasets in full.

\section{References}

1. Charles AS, Falk B, Turner N, Pereira TD, Tward D, Pedigo BD, et al. Toward Community-Driven Big Open Brain Science: Open Big Data and Tools for Structure, Function, and Genetics. Annu Rev Neurosci. 2020;43: 441-464.

\footnotetext{
${ }^{1}$ This term was coined by Fernando Perez to describe the dynamics that occur around large, valuable, publicly available datasets (Personal Communication).
} 
2. Mallory CS, Hardcastle K, Campbell MG, Attinger A, Low IIC, Raymond JL, et al. Recordings from medial entorhinal cortex during linear track and open exploration. Mouse entorhinal cortex encodes a diverse repertoire of self-motion signals. 2021.

doi:https://dandiarchive.org/dandiset/000053/draft

3. Glasser MF, Smith SM, Marcus DS, Andersson JLR, Auerbach EJ, Behrens TEJ, et al. The Human Connectome Project's neuroimaging approach. Nat Neurosci. 2016;19: 1175-1187.

4. Alexander LM, Escalera J, Ai L, Andreotti C, Febre K, Mangone A, et al. An open resource for transdiagnostic research in pediatric mental health and learning disorders. Sci Data. 2017;4: 170181.

5. Jernigan TL, Brown SA, Dowling GJ. The Adolescent Brain Cognitive Development Study. J Res Adolesc. 2018;28: 154-156.

6. Sudlow C, Gallacher J, Allen N, Beral V, Burton P, Danesh J, et al. UK biobank: an open access resource for identifying the causes of a wide range of complex diseases of middle and old age. PLoS Med. 2015;12: e1001779.

7. Marek S, Tervo-Clemmens B, Calabro FJ, Montez DF, Kay BP, Hatoum AS, et al. Towards Reproducible Brain-Wide Association Studies. 2020. p. 2020.08.21.257758. doi:10.1101/2020.08.21.257758

8. Downs BN, Opheim DM, Hale W, Xi L, Donehower LA, Kalra D. A Practical Example of Bringing Computation to Data. J Biomol Tech. 2014;25: S5.

9. Zaharia M, Borthakur D, Sarma JS. Delay scheduling: A simple technique for achieving locality and fairness in cluster scheduling. [cited 17 Feb 2021]. Available: https://cs.stanford.edu/ matei/papers/2010/eurosys_delay_scheduling.pdf

10. Kiar G, Gorgolewski KJ, Kleissas D, Roncal WG, Litt B, Wandell B, et al. Science in the cloud (SIC): A use case in MRI connectomics. Gigascience. 2017;6: 1-10.

11. Bommadevara N, Del Miglio A, Jansen S. Cloud adoption to accelerate IT modernization. McKinsey \& Company; 10 Apr 2018 [cited 18 Aug 2021]. Available: https://www.mckinsey.com/business-functions/mckinsey-digital/our-insights/cloud-adopti on-to-accelerate-it-modernization

12. Pérez F, Hamman J, Larsen L, Paul K, Heagy L, Holdgraf C, et al. Jupyter meets the Earth: Enabling discovery in geoscience through interactive computing at scale. 2019. doi:10.5281/zenodo.3369939

13. Kluyver T, Ragan-Kelley B, Pérez F, Granger B, Bussonnier M, Frederic J, et al. Jupyter Notebooks-a publishing format for reproducible computational workflows. Positioning and Power in Academic Publishing: Players, Agents and Agendas. 2016; 87.

14. Behrens JT. Principles and procedures of exploratory data analysis. Psychol Methods. 2: 131-160.

15. Gelman A. Exploratory Data Analysis for Complex Models. J Comput Graph Stat. 2004;13: 755-779. 
16. Granger B, Pérez F. Jupyter: Thinking and storytelling with code and data. Authorea, Inc.; 2021. doi:10.22541/au.161298309.98344404/v2

17. Rocklin M. Dask: Parallel Computation with Blocked algorithms and Task Scheduling. In: Huff K, Bergstra J, editors. Proceedings of the 14th Python in Science Conference. 2015. pp. 126-132.

18. Brewer EA. Kubernetes and the path to cloud native. Proceedings of the Sixth ACM Symposium on Cloud Computing. New York, NY, USA: Association for Computing Machinery; 2015. p. 167.

19. Gorgolewski KJ, Auer T, Calhoun VD, Craddock RC, Das S, Duff EP, et al. The brain imaging data structure, a format for organizing and describing outputs of neuroimaging experiments. Sci Data. 2016;3: 160044.

20. Gramfort A, Luessi M, Larson E, Engemann DA, Strohmeier D, Brodbeck C, et al. MEG and EEG data analysis with MNE-Python. Front Neurosci. 2013;7: 267.

21. Pedregosa F, Varoquaux G, Gramfort A, Michel V, Thirion B, Grisel O, et al. Scikit-learn: Machine Learning in Python. arXiv [cs.LG]. 2012. Available: http://arxiv.org/abs/1201.0490

22. Kruper J, Yeatman JD, Richie-Halford A, Bloom D, Grotheer M, Caffarra S, et al. Evaluating the reliability of human brain white matter tractometry. bioRxiv. 2021. p.

2021.02.24.432740. doi:10.1101/2021.02.24.432740

23. Richie-Halford A, Rokem A. Cloudknot: A python library to run your existing code on aws batch. Proceedings of the 17th python in science conference. 2018. pp. 8-14.

24. Holdgraf C, Culich A, Rokem A, Deniz F, Alegro M, Ushizima D. Portable Learning Environments for Hands-On Computational Instruction: Using Container- and Cloud-Based Technology to Teach Data Science. Proceedings of the Practice and Experience in Advanced Research Computing 2017 on Sustainability, Success and Impact. New York, NY, USA: Association for Computing Machinery; 2017. pp. 1-9.

25. Gau R, Noble S, Heuer K, Bottenhorn KL, Bilgin IP, Yang Y-F, et al. Brainhack: Developing a culture of open, inclusive, community-driven neuroscience. Neuron. 2021.

doi:10.1016/j.neuron.2021.04.001

26. Cameron Craddock R, S Margulies D, Bellec P, Nolan Nichols B, Alcauter S, A Barrios F, et al. Brainhack: a collaborative workshop for the open neuroscience community. Gigascience. 2016;5: 16.

27. Abernathey RP, Augspurger T, Banihirwe A, Blackmon-Luca CC, Crone TJ, Gentemann CL, et al. Cloud-Native Repositories for Big Scientific Data. Computing in Science Engineering. 2021;23: 26-35.

28. Miles A, jakirkham, Bussonnier M, Durant M, Moore J, Fulton A, et al. zarr-developers/zarr-python: v2.8.3. 2021. doi:10.5281/zenodo.5091549

29. Hoyer S, Hamman J. xarray: ND labeled arrays and datasets in Python. Journal of Open Research Software. 2017;5. Available: 
https://openresearchsoftware.metajnl.com/articles/148/

30. Wilkinson MD, Dumontier M, Aalbersberg IJJ, Appleton G, Axton M, Baak A, et al. The FAIR Guiding Principles for scientific data management and stewardship. Sci Data. 2016;3: 160018.

31. Keshavan A, Datta E, M McDonough I, Madan CR, Jordan K, Henry RG. Mindcontrol: A web application for brain segmentation quality control. Neuroimage. 2017. doi:10.1016/j.neuroimage.2017.03.055

32. Dougherty RF, Koch VM, Brewer AA, Fischer B, Modersitzki J, Wandell BA. Visual field representations and locations of visual areas V1/2/3 in human visual cortex. J Vis. 2003;3: 586-598.

33. Hagmann $P$, Thiran J-P, Jonasson L, Vandergheynst $P$, Clarke S, Maeder $P$, et al. DTI mapping of human brain connectivity: statistical fibre tracking and virtual dissection. Neuroimage. 2003;19: 545-554.

34. Catani M, Howard RJ, Pajevic S, Jones DK. Virtual in vivo interactive dissection of white matter fasciculi in the human brain. Neuroimage. 2002;17: 77-94.

35. Yeatman JD, Richie-Halford A, Smith JK, Keshavan A, Rokem A. A browser-based tool for visualization and analysis of diffusion MRI data. Nat Commun. 2018;9: 940.

36. Sherif T, Kassis N, Rousseau M-É, Adalat R, Evans AC. BrainBrowser: distributed, web-based neurological data visualization. Front Neuroinform. 2014;8: 89.

37. Amunts K, Lepage C, Borgeat L, Mohlberg H, Dickscheid T, Rousseau M-É, et al. BigBrain: an ultrahigh-resolution 3D human brain model. Science. 2013;340: 1472-1475.

38. Maitin-Shepard J. neuroglancer. Github. 2019.

39. Robitaille T, Beaumont C, Qian P, Borkin M, Goodman A. glueviz v0.13.1: multidimensional data exploration. 2017. doi:10.5281/zenodo.1237692

40. Turk MJ, Smith BD, Oishi JS, Skory S, Skillman SW, Abel T, et al. yt: A MULTI-CODE ANALYSIS TOOLKIT FOR ASTROPHYSICAL SIMULATION DATA. ApJS. 2010;192: 9.

41. Bednar J, Lead OST, Senior Solutions Architect A. Big Data Visualization With Datashader. Anaconda, Inc : Austin, TX, USA. 2017; 12.

42. Vogelstein JT, Perlman E, Falk B, Baden A, Gray Roncal W, Chandrashekhar V, et al. A community-developed open-source computational ecosystem for big neuro data. Nat Methods. 2018;15: 846-847. 